\title{
ESTUDIO DE LA INCIDENCIA DE UNA PISCIFACTORIA EN LAS COMUNIDADES DE MACROINVERTEBRADOS ACUATICOS
}

\author{
A. Domezain, I. Guisasola y J. Alba-Tercedor \\ Departamento de Biología Animal, Ecología y Genética; (Zoología), Facultad de Ciencias (Biológicas), Universi- \\ dad de Granada, 18001 Granada.
}

Palabras clave: Fishfarm, macroinvertebrates, biotic index.

\section{ABSTRACT \\ STUDY OF THE EFFECT OF A TROUTFARM ON THE AQUATIC MACROINVERTEBRA- TE COMMUNITIES}

\begin{abstract}
The effect of a troutfarm on the rnacroinvertebrate populations of a strearn was studied and sorne biotic indices were used. It was concluded that the effect altought slight was clearly detected by the macroinvertebrates,increasing or decreasing their populations. Likewise a habitat transposition was observed.

On the other hand both E.B.I. (Woodiwiss,1978) and Hellawell's (1978)index were most suitable for testing the fishfarm effect on the stream dueto the good water quality interpretation ofthe first one and easy use and sensivity on the second.
\end{abstract}

\section{INTRODUCCION}

La proximidad a nuestro laboratorio de una de las piscifactorías más antiguas y de mayor producción de truchas del sur de la Península (400 Tm./año), asî como la polémica que este tipo de instalaciones despierta sobre las posibles alteraciones que originan en los cursos de agua, nos motivó a estudiar los efectos de la misma en las poblaciones de macroinvertebrados acuáticos.

\section{MATERIAL Y METODOS}

El estudio se realizó en la piscifactoría situada en la margen derecha del río Frío (afluente del Genil), aguas abajo del núcleo urbano de Río Frío (pequeña población de unos 400 habitantes), situado en el término municipal de Loja (prov. de Granada) a $580 \mathrm{~m}$. de altitud, en el punto de coordenadas U.T.M.: 30SUG9313 que presenta una pendiente del 1\%. Durante los Ultimos años el personal de la piscifactoría, utilizando un molinete hidráulico midió unos caudales que oscilaron entre 0,7 y $3 \mathrm{~m} .{ }^{3} / \mathrm{seg}$., registrando hasta $100 \mathrm{~m} .{ }^{3} / \mathrm{seg}$., en condiciones de riada. Según la misma fuente la temperatura a lo largo del

Limnética 3: 151-157 (1987)

(c) Asociación Española de Limnología, Madrid. Spain año es muy constante, generalmente de $15^{\circ} \mathrm{C}$; oscilando entre 13.5 y $19^{\circ} \mathrm{C}$.

El agua utilizada por la piscifactoría pasa por dos fosas de decantación, no comunicadas entre sí, antes de devolverse al cauce del río, existiendo dos puntos de vertido independientes, separados $100 \mathrm{~m}$., cada uno de los cuales procede de una fosa de decantación.

Para llevar a cabo nuestros objetivos, a comienzos de diciembre de 1983 muestreamos las poblaciones de macroinvertebrados en cuatro puntos diferentes. Punto I: situado algunos metros aguas arriba de las instalaciones de la piscifactoría; punto II: $250 \mathrm{~m}$. aguas abajo del primero, en el primer punto de vertido; punto III: $100 \mathrm{~m}$. aguas abajo del segundo, justo en el lugar en que se produce el segundo vertido; punto IV: situado $500 \mathrm{~m}$. aguas abajo del último vertido de la piscifactoría.

La captura de los macroinvertebrados se realizó con una red con abertura triangular de 40 x $26 \mathrm{~cm}$. y un tamaño de malla de $0,36 \mathrm{~mm}$. Se distinguió entre zonas de corriente rápida (aguas lóticas) y zonas de escasa corriente (aguas Iénticas) y dentro de estas últimas se separaron las zonas de fondos más o menos lodosos de las de vegetación, guijarros o piedras. Además, en los puntos II y IV se muestrearon por separado las masas de musgos existentes. 


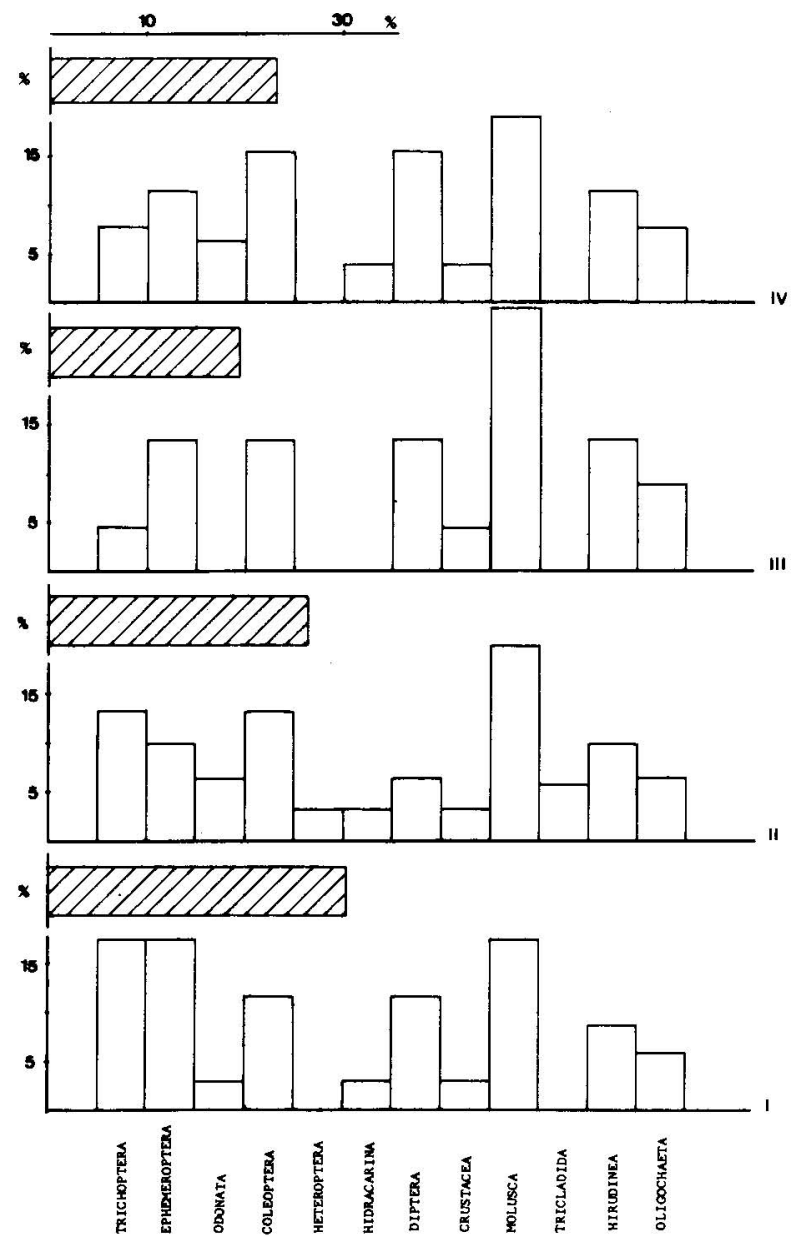

Figura 1.- Porcentaje de unidades sistemáticas consideradas presentes en cada una de las cuatro estaciones de muestreo. Las barras horizontales rayadas representan el númerototal de taxones presentes en cada estación de muestreo.

Percentage of the systematic unities considered to be present in each of the four sampling stations. The horizontal crosshatchedbars represent the total number of taxons that occur in each sampling station.

El método de muestreo consistía en que mientras un investigador sujetaba la red, otro removía y frotaba todo el sustrato situado inmediatamente aguas arriba, repitiéndose la operación hasta cubrir una longitud de unos $2 \mathrm{~m}$. y esto para cada una de las facies estudiadas.

El contenido total de cada redada se guardaba en frascos a los que se añadía agua y formol hasta alcanzar una concentración del 7\%. Posteriormente, en el laboratorio, se extrajeron los macroinvertebrados conservándolos en alcohol al $70 \%$.

Tras la identificación y recuento de los organismos se estudió la calidad biológica de las aguas en base a la aplicación de los siguientes índices de calidad: T.V. (Tuffery \& Verneaux, 1967), E.B.I. (Woodiwiss,
1978), B.S. (Chandler, 1970) y Hellawell (1978) (Según Armitage et al., 1983).

\section{RESULTADOS}

En la tabla 1 se relacionan los macroinvertebrados capturados en cada punto de muestreo, según diferentes facies del lecho, indicándose el número de individuos de cada uno de los taxones considerados.

En base a los datos anteriores se construyeron histogramas para visualizar el peso porcentual de los grandes grupos de macroinvertebrados en cada uno de los puntos de muestreo. Para ello se tuvieron en cuenta tan sólo las unidades sistemáticas utilizadas 


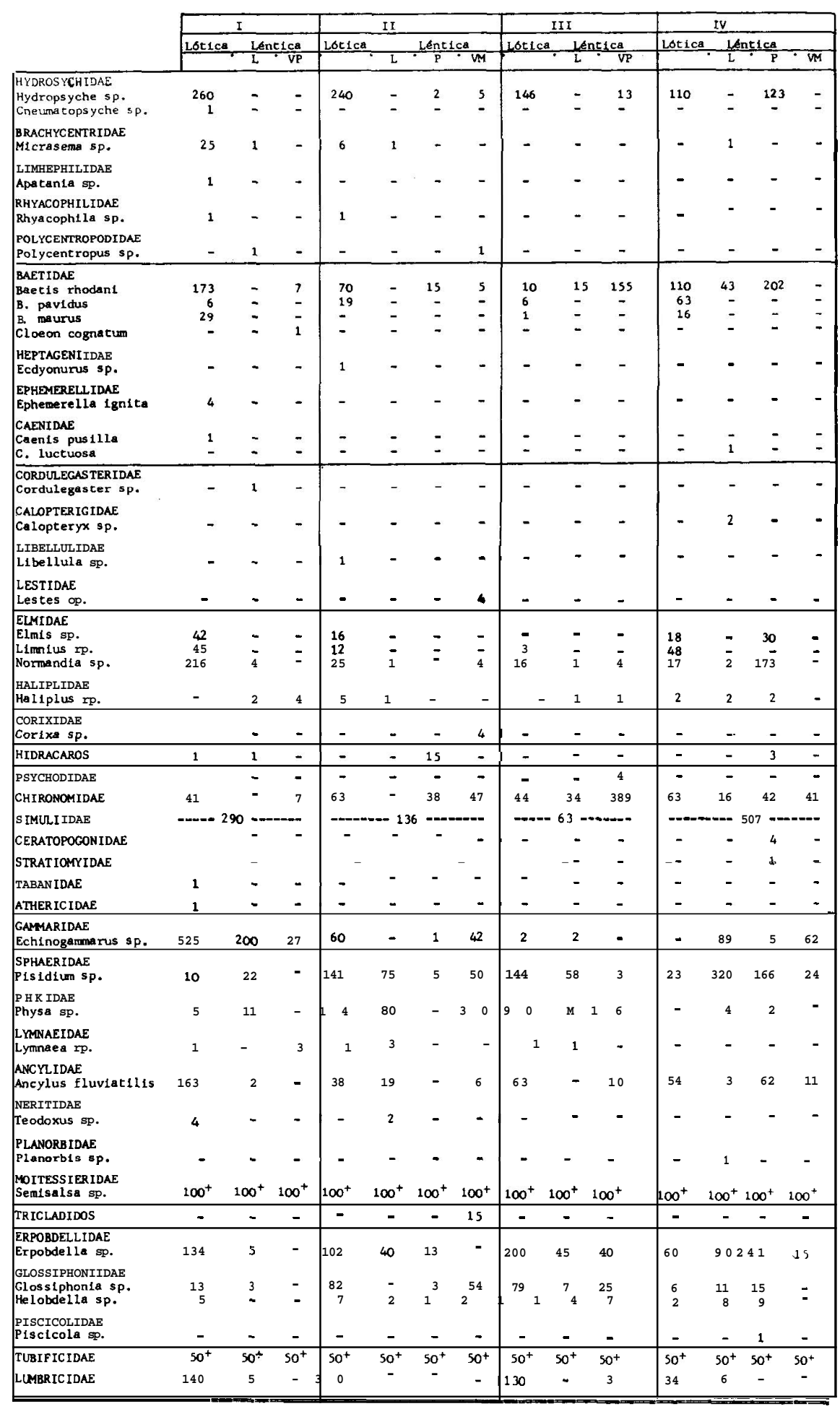

Tabla 1.- Macroinvertebrados capturados en las diferentes estaciones de muestreo.

Macroinvertebrates caught in each sampling stations. 

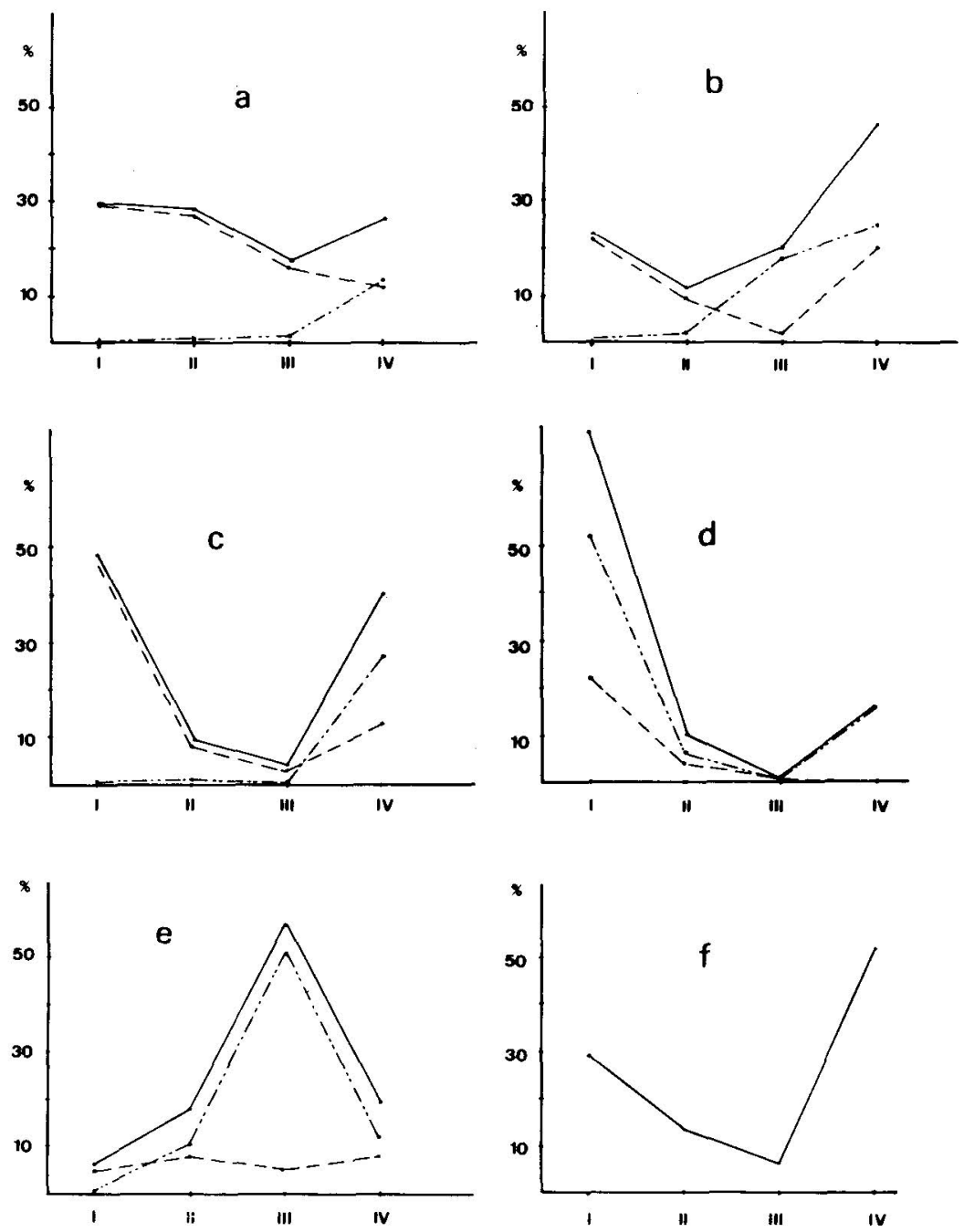

Figura 2.- Porcentaje del número total de individuos de algunos taxones de macroinvertebrados presentes en cada estación de muestreo: a) Hydropsyche sp.; b) Baetis sp.; c) Elmidae; d) Echinogammarus sp.; e) Chironomidae; f) Simulidae. (Con rayas discontinuas se representan los individuos capturados en zonas lóticas; con rayas y puntos los capturados en zonas lénticas y con línea continua el total de individuos capturados).

Percentage of the total number of individuals of some macroinvertebrates taxons present in each sampling station: a) Hydropsyche sp.; b) Baetis sp.; c) Elmidae; d) Echinogammarus sp.; e) Chironomidae; f) Simulidae. (Discontinuous lines represent individuals caught in lotical zones; lines and points represent individuals caught in lentical zonae and with continous lines the whole of individuals caught were represented).

para la obtención de los índices de T.V. y E.B.I. (familias en: Tricópteros, Coleópteros, Crustáceos, Dípteros y Oligoquetos; géneros en: Efemerópteros, Odonatos, Moluscos, Heterópteros Tricládidos e Hirudínidos; y la mera presencia en el resto) (Ghetti \& Bonazzi, 1981). De este modo en la fig. 1 se puede apreciar en barras horizontales rayadas el porcentaje del total de unidades sistemáticas en cada uno de los puntos de muestreo en relación a la suma de unidades sistemáticas existentes en cada uno de los puntos de muestreo y en barras verticales se representa el porcentaje de unidades sistemáticas de cada uno de los grandes grupos de macroinvertebrados, en relación al total de unidades sistemáticas presentes en el punto de muestreo considerado.

Por otro lado en las figuras 2 y $\mathbf{3}$ se muestran las 

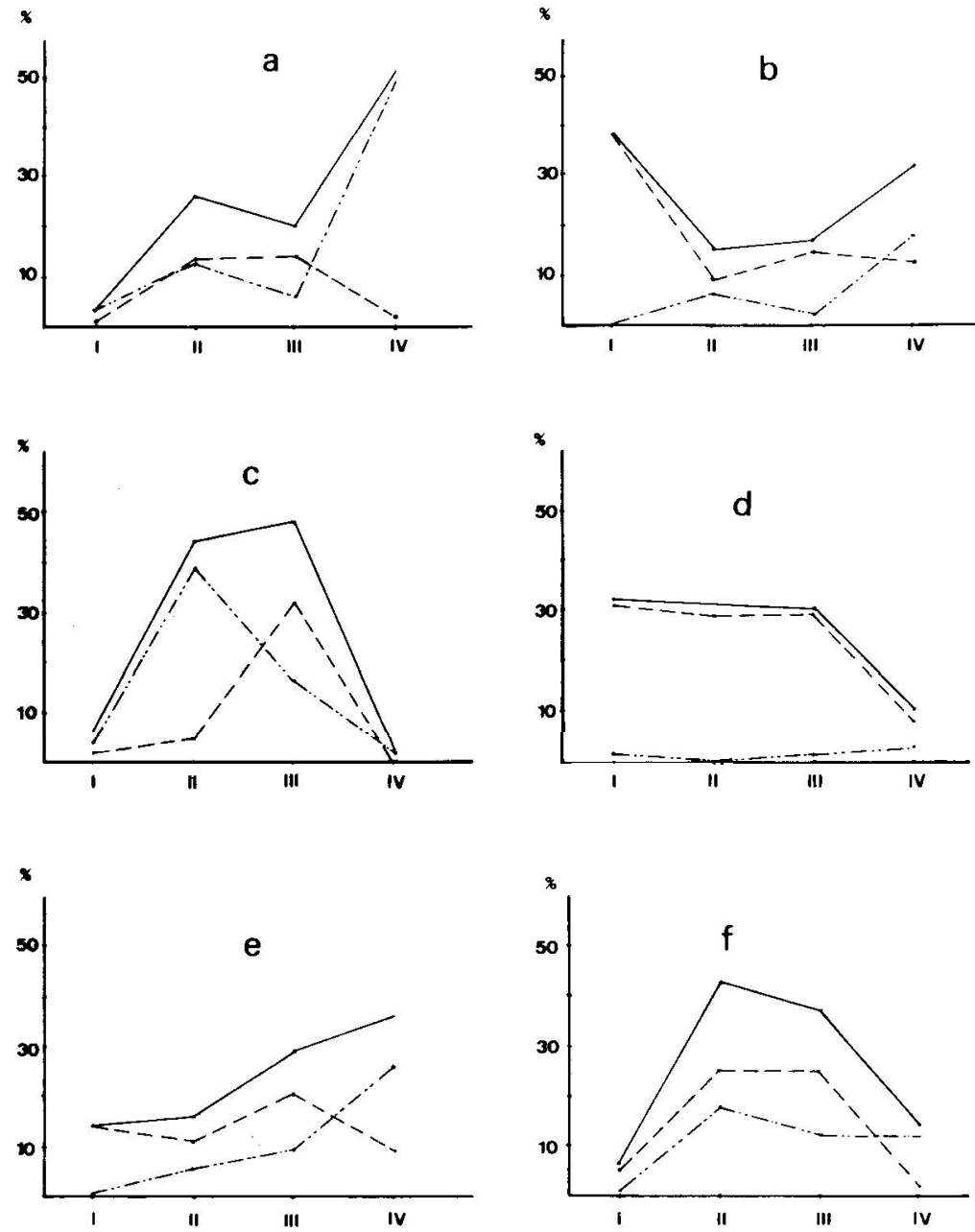

Figura 3.- Porcentaje del número total de individuos de algunos taxones de macroinvertebrados presentes en cada estación de muestreo: a) Pisidium sp.; b) Ancylus fluviatilis; c) Physa sp.; d) Lumbricidae; e) Erpobdella sp.; f) Glossiphonia sp. (Con rayas discontinuas se representan los individuos capturadosen zonas lóticas; con rayas y puntos los capturados en zonas lénticas y con línea continua el total de individuos capturados).

Percentage of the total number of individuals of some macroinvertebrates' taxons present in each sampling station:a) Pisidium sp.; b) Ancylus fluviatilis; c) Physa sp; d) Lumbricidae;e) Erpobdella sp.; f) Glossiphonia sp.; (Discontinouslines represent individuals caught in lotical zones; lines and points represent individuals caught in lentical zones and with continous lines the whole of individuals caught were represented).

respuestas de determinados organismos a los sucesivos vertidos, expresadas en función del porcentaje del numero total de individuos presentes en cada taxón.

Por último se han representado las variaciones de los diferentes índices biológicos en los puntos de muestreo (figs. 4 y 5). En la fig. 4 se expresan los índices en \%; para ello se consideró que el $100 \%$ de cada índice correspondería con valores de : 10, 12 y 2.000 respectivamente para los índices de T.V., E.B.I. y BS. (basándonos para este último en Ghetti \& Bonazzi, 1981). En el caso del índice de Hellawell (1978), al ser (al igual que el BS) un índice abierto por arriba y no disponer de datos sobre máximos que alcanza, lo hicimos coincidir en el punto I con el valor porcentual calculado para el BS. y en función de este calculamos el equivalente para los otros puntos. La fig. 5 muestra la variación en la calidad biológica del agua de los puntos de muestreo, dentro de las cinco clases de calidad que admiten el E.B.I. y el índice de T.V. 

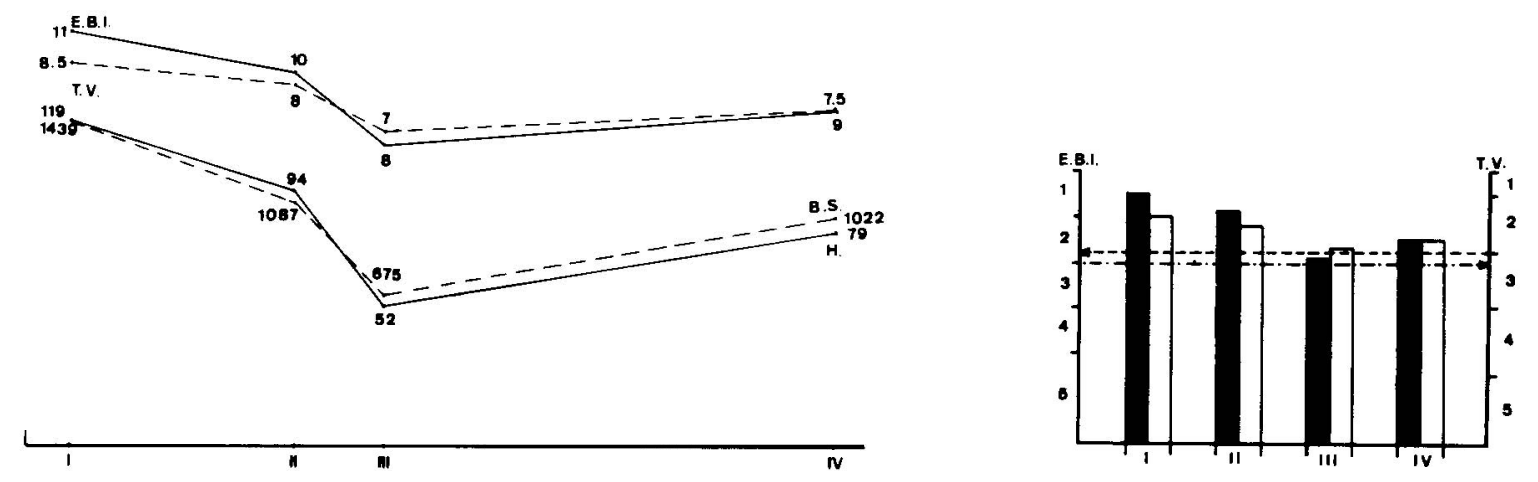

Figura 4.- Valores porcentuales de diferentes índices bióticos en cada estación de muestreo. Se indican los valores calculados para cada índice. (Verexplicación en el texto).

Percentage values of different biotic indexes in each sampling station. The values calculated for each index are indicated. (See explanation in the text).

Figura 5.- Calidad biológica del agua en las diferentes estaciones de muestreo, basada en las cinco clases de calidad de los indices de Woodiwiss (1978)(E.B.I.)y Tuffery y Vemeaux (1967)(T.V.).(En sombreado se indican los valores de E.B.I.).

Water biological quality in each sampling station based on the five quality ranks of the Woodiwiss (1978)(E.B.I.)and Tuffery \& Vemeaux (1967) (T.V.)indexes. (The shaded bars represent the E.B.I. values).

\section{DISCUSION}

Los vertidos de la piscifactoría son claramente detectados por la biocenosis acuática, lo que se traduce en una disminución del número de unidades sistemáticas consideradas y en variaciones en el número de individuos de los diferentes taxones aguas abajo de los sucesivos vertidos (Tabla 1 y figs.: 1-3) que a su vez se refleja en unas disminuciones de los valores de los índices bióticos (fig. 4); si bien la calidad biológica del agua se mantuvo siempre dentro de unos límites aceptables (fig. 5).

Al realizar el muestreo, habíamos apreciado que aguas abajo de los vertidos el lecho del Río presentaba un sensible incremento de sedimentos orgánicos; observándose en nuestros resultados una clara respuesta de los macroinvertebrados, bien por una disminución en sus poblaciones (fig. 2: a, c, d y f), bien por un incremento en las mismas (figs. $2 \mathrm{e}, 3 \mathrm{c}, 3 \mathrm{e}, 3 \mathrm{f}$ ), respondiendo a necesidades ecológicas y grados de tolerancia bien conocidos para los distintos grupos (Echaubard \& Neveu, 1975; Hart $\boldsymbol{e t}$ al., 1974; Hynes, 1960; Tuffery, 1976; Wiederholm, 1984). Por otro lado y a medida que aumenta el grado de alteración, se produce un desplazamiento de los organismos hacia las zonas lóticas en detrimento de las Iénticas (fig. 3: a,b,c,e,f), hecho observado por otros autores (Verneaux, 1976).

Respecto a la interpretación de la calidad biológica de las aguas en función de los índices de T.V. y E.B.I. (Ghetti \& Bonazzi, 1981), los resultados de uno y otro concuerdan en señalar que la contaminación es leve (la clase más baja fue de 2) (fig. 5), si bien el E.B.I. resultó más preciso que el índice de T.V.:

a) Punto I.-Según el indice de T.V. las aguas de que se surte la piscifactoría son de calidad «aceptable» (clase 2), si bien el valor 8.5 de este indice es interpretado por sus autores como indicador de que existe una ligera contaminación. Mientras que segun E.B.I. en ese punto o no hay contaminación presente o si la hay es muy leve (clase 1). De hecho el Río recibe los vertidos de la pequeña población de Río Frío y de establecimientos hoteleros situados inmediatamente aguas arriba de la piscifactona, además de una almazara situada $1.5 \mathrm{kms}$. aguas arriba, cuyos efectos en las comunidades de macroinvertebrados fueron puestos de manifiesto por Vilchez (1982).

b) Punto II.- Los valores de los índices bióticos disminuyen en cierta medida. Sin embargo tanto el E.B.I. como el índice de T.V. permanecen dentro de las mismas clases de calidad (Figs. 4 y 5).

c) Punto III.- Es en este punto donde todos los índices alcanzan los valores mínimos (Fig. 4) y tanto a la luz del E.B.I. (Clase 2) como del índice de T.V. (Clase 2), (clase 2), en el ambiente son evidentes algunos efectos de la contaminación.

d) Punto IV.- Se observa una clara tendencia a la recuperación de los valores de los índices bióticos (fig. 4), si bien ésta no es total, de tal modo que según los índices de T.V. y E.B.I. se mantiene la calidad del agua dentro de la clase 2 (fig. 5). Es decir, una calidad aceptable en la que de hecho (al igual que en el punto 
anterior) se desarrollan muy bien tanto la trucha arcoiris (Salmo gaidnieri) como la endémica trucha fario (Salmo trutta fario).

De todo lo anterior se deduce que si bien la piscifactoría altera el Río al verter las aguas utilizadas, esta alteración (según la interpretación de calidad del agua basada en las variaciones de los valores de los índices que establecen Tuffery \& Verneaux, 1967 y Woodiwiss 1978) es leve, observándose una clara tendencia a la recuperación $500 \mathrm{~m}$. aguas abajo del último punto de vertido (figs. 1 a 4 ). Desde el punto de vista de la interpretación de la calidad biológica de las aguas el índice de Tuffery \& Verneaux (1967) resultó «menos preciso» que el de Woodiwiss (1978), debido a que según el índice de T.V. la calidad se mantuvo en clase 2, en todo momento; mientras que por el contrario el E.B.I. discriminó entre las situaciones de antes de los vertidos (clase 1) y después de los mismos (clase 2) (fig. 5). Además si bien todos los índices utilizados detectaron claramente los sucesivos vertidos, los índices más sensibles resultaron ser el de Chandler (1970) y Hellawell (1978) (fig. 4). Lo que unido a que mientras que el índice de Chandler (1970) exige para su aplicación un muestreo semicuantitativo, la identificación de los organismos en muchos casos a nivel específico y recuentos del número de ejemplares de cada taxón, con el tiempo que ello supone; por el contrario el índice de Hellawell (1978) no exige más que un muestreo cualitativo y una identificación tan solo a nivel de familia. Todo ello sugiere la utilidad práctica de éste último índice para el seguimiento de los efectos de este tipo de instalaciones en los cursos de agua.

\section{BIBLIOGRAFIA}

Armitage, P.B., Moss, D., Wright, J.F. \& Furse, M.T., 1983. The performance of a new biological water quality score system based on macroinvertebrates over a wide range of unpolluted running-water sites. Water Res., 17(3): 333-347.
Chandler, J.R. 1970. A biological approach to water quality management Wat. Pollut. Control. London., 69: 415-422.

Echaubard, M. \& Neveu, A. 1974. Perturbations qualitatives de la faune benthique d'un ruisseau a truites, La Couze Pavin (Puy-de Dome), dues aux pollutions agricoles et urbaines. Ann. Stn. Biol. Besse-en-Chandesse, 9/1-24.

Ghetti, P.F. \& Bonazzi, G. 1981. I macroinvertebrati nella sorveglianza ecologica dei corsi dácqua. Promozione della qualitá dell'ambiente, $\mathrm{AQ} / 1 / 127 /$. Roma, 181 págs.

Hart, Jr. C.W., \& Fuller, S.L.H. (Eds.) 1974. Pollution Ecology of Freshwater Invertebrates. Academic Press. New York, S. Francisco and London, 389 págs.

Hellawell, J.M. 1978. Biological surveillance of rivers. Water Research Centre, Stevenage, 332 págs.

Hynes, H.B.N. 1960. The biology of polluted waters. Liverpool University Press, Liverpool, 202 págs.

Tuffery, G. 1976. Incidencias ecológicas de la polución de las aguas corrientes. Reveladores biológicos de la polución. En: La contaminación de las aguas corrientes continentales (Pesson, P. ed.) 1979: 215-255. Mundi Prensa. Madrid. XXXVII + 331 págs.

Tuffery, G. \& Verneaux, J. 1967. Méthode de détennination de la qualité biologique des eaux courantes. Exploitation codifié des inventaires de la faune de fond. Trav. Sect. Tech. P. et P., C.E.R.A.F.E.R., París, 23 págs.

Verneaux, J. 1976. Fundamentos biológicos y ecológicos del estudio de la calidad de las aguas continentales. Principales métodos biológicos. En: La contaminación de las aguas continentales (Pesson, P. ed.) 1979: 267-331 . Mundi Prensa. Madrid. XXXVII+ 331 págs.

Vilchez Quero, A. 1982. Efecto de la contaminación orgánica sobre las poblaciones benticas de macroinvertebrados de los ríos Frío y Cubillas (Granada).Con especial referencia a Dipteros Quironómidos. Tesis doctoral (inédita),Univ. de Granada, 320 págs.

Wiederholrn, T. 1984. Responses of Aquatic Insects to Environmental Pollution. En: The Ecology of Aquatic Insects (Resh, V.H. and Rosenberg, D.M. eds.): 508-557. Praeger Publishers. New York, IX + 625 págs.

Woodiwiss, F.S. 1978. Biological Water Assessment Methods. Sevem-Trent River Authorities, U.K. 